\title{
A Technique for the Rapid Selection of Drug-sensitive and Auxotrophic Mutants of Phycomyces blakesleeanus
}

\author{
By KAREN J. BRUNKE, R. MERCURIO AND S. H. GOODGAL \\ Department of Microbiology, School of Medicine, University of Pennsylvania, \\ Philadelphia, Pennsylvania 19174, U.S.A.
}

(Received 7 March 1977)

\begin{abstract}
An auxotroph enrichment procedure has been developed for Phycomyces blakesleeanus which has proven useful in obtaining both auxotrophs and drug-sensitive mutants. The technique is based on the differential heat sensitivity between ungerminated auxotrophic spores and germinated prototrophic spores. Germinated spores and mycelia die when left at temperatures higher than about $35^{\circ} \mathrm{C}$ for 16 to $24 \mathrm{~h}$ but ungerminated spores survive this treatment and subsequently germinate if transferred to complete medium. With the dwarf colonial strain, replica plating permits the rapid characterization of these newly selected auxotrophs.
\end{abstract}

\section{INTRODUCTION}

In various methods devised to increase the proportion of auxotrophic mutants in a population (Moat, Peters \& Srb. 1959; Snow, I 966; Henry, 1973), the underlying principle is the creation of conditions where non-growing auxotrophs survive while growing prototrophic cells are destroyed. In fungi, auxotrophic mutants can be isolated by modified bacterial techniques. In yeast, for example (Megnet, 1964; Ridgway \& Douglas, 1958), certain inositol-requiring strains, Inos-, die in its absence. However, the occurrence of a second auxotrophic mutation prevents growth thus avoiding inositol-less death. By using Inosstrains treated with a mutagen, it is possible to increase the proportion of new auxotrophs in the population. Replica-plating (Lederberg \& Lederberg, 1952) allows auxotrophs to be isolated and characterized rapidly.

Without similar procedures in Phycomyces blakesleeanus, mutant selection has been very difficult. A minimal medium liquid culture-filtration procedure (S. Park, unpublished results; Woodward, De Zeeuw \& Srb, 1954) has facilitated auxotroph enrichment in Phycomyces. Only prototrophs grow in the minimal medium while ungerminated, presumably auxotrophic, spores can be separated from these by filtration. Using acid agar conditions to permit cloning of spores (S. Park, unpublished results), auxotrophs can then be isolated and characterized. The isolation in this laboratory, after ultraviolet (u.v.) light mutagenesis, of a mutant strain of Phycomyces with dwarf sporangiophores and a colonial habit (W. L. Hom, unpublished results) has allowed the use of replica-plating as a rapid means of examining individual clones for drug resistance, drug sensitivity and auxotrophic mutations.

Although liquid culture-filtration selection has been successful with Phycomyces in enriching for auxotrophs, repeated cycles were necessary to eliminate prototrophs. Mutagenic treatment apparently delayed cell division in a treated population so that differences between prototrophs and auxotrophs developed only after several selective cycles. Even when spores failed to germinate in a minimal medium liquid culture, they would often appear on minimal medium agar plates, so a better selection technique was desirable.

In the studies reported here, the development of a new technique involving the differential 
heat sensitivity of spores and mycelia has speeded selection of auxotrophs in a search for mutants of the dwarf colonial strain and selection of drug-sensitive mutants from an erythromycin-resistant strain to be used as recipients in organelle transfer studies (Goodgal, Kahn \& Keyhani, 1975). A variety of stable auxotrophs were obtained over a short period using this technique. Auxotrophic strains previously isolated, but in which a low percentage of spores would grow without one or more supplements, were easily purified by differential heat selection.

\section{METHODS}

Strains. Two strains of Phycomyces blakesleeanus were used to obtain mutants: strain P8, a nicotinic acid auxotroph obtained in this laboratory from the wild-type strain NRRLI554 (+); and strain PII (col-5I $d w f-I r i b-I)$, a riboflavin-requiring dwarf colonial mutant isolated in this laboratory following u.v.-irradiation of spores from the wild-type strain NRRLI555 (-).

Media and growth conditions. Minimal medium for agar plates contained: $\mathrm{I} \cdot 5 \%(\mathrm{w} / \mathrm{v})$ Difco agar; $3 \%$ (w/v) glucose; $2.0 \mathrm{mg}$ asparagine $\mathrm{ml}^{-1} ; 1.5 \mathrm{mg} \mathrm{KH}_{2} \mathrm{PO}_{4} \mathrm{ml}^{-1} ; 0.5 \mathrm{mg} \mathrm{MgSO}_{4} \mathrm{ml}^{-1}$; and $0.5 \mu \mathrm{g}$ thiamin $\mathrm{ml}^{-1}$. Minimal medium for liquid cultures was identical except for the omission of agar. Enriched complete medium for agar plates contained: $5 \%(\mathrm{w} / \mathrm{v})$ potato dextrose agar; $0.5 \%(\mathrm{w} / \mathrm{v})$ peptone; and $0.5 \%(\mathrm{w} / \mathrm{v})$ yeast extract. Complete medium for liquid cultures contained: $0.5 \%$ yeast extract; $0.5 \%$ peptone; $3 \%$ glucose; $2.0 \mathrm{mg}$ asparagine $\mathrm{ml}^{-1} ; \mathrm{I} .5 \mathrm{mg} \mathrm{KH}_{2} \mathrm{PO}_{4} \mathrm{ml}^{-1} ; 0.5 \mathrm{mg} \mathrm{MgSO}_{4} \mathrm{ml}^{-1}$; and $0.5 \mu \mathrm{g}$ thiamin $\mathrm{ml}^{-1}$. Penicillin ( $\mathrm{mg} \mathrm{ml}^{-1}$; potassium penicillin G, U.S.P.; E. R. Squibb, New Brunswick, New Jersey, U.S.A.) was added to all media to prevent bacterial contamination. Nicotinic acid $\left(\mathrm{I} \mu \mathrm{g} \mathrm{m}^{-1}\right)$ was added to minimal medium for growth of strain P8, and riboflavin $\left(\mathrm{I} \mu \mathrm{g} \mathrm{ml}^{-1}\right)$ for growth of strain PI I.

All spores were heat-shocked for to to $15 \mathrm{~min}$ at $48{ }^{\circ} \mathrm{C}$ after inoculation to ensure maximum germination. Liquid cultures were grown for 20 to $48 \mathrm{~h}$ at room temperature with aeration.

Antibiotics. For testing erythromycin sensitivity, solutions of erythromycin (sterile erythromycin gluceptate, U.S.P.; Eli Lilly, Indianapolis, Indiana, U.S.A.) were made in sterile distilled water; these were kept refrigerated and used within 7 days.

Materials. The dialysis tubing (Arthur H. Thomas, Philadelphia, Pennsylvania, U.S.A.) used in transfers between plates had a molecular weight cut-off of 8000 and was $9.2 \mathrm{~cm}$ in diameter with a wall thickness of $0.09 \mathrm{~mm}$.

Mutagenesis. Spores were heat-shocked at $48^{\circ} \mathrm{C}$ for $15 \mathrm{~min}$ and then sedimented to give pellets containing $10^{7}$ to $10^{8}$ spores as determined in a Petroff-Hauser counter. The spore pellets were resuspended in $0.5 \mathrm{ml}$ of $0.2 \mathrm{M}$-sodium acetate buffer $\mathrm{pH} 5.0$, and $0.5 \mathrm{ml}$ of a fresh solution of $N$-methyl- $N^{\prime}$-nitro- $N$-nitrosoguanidine ( $1 \mathrm{mg} \mathrm{ml}^{-1}$; NTG; K \& K Laboratories, Plainview, New York, U.S.A.) was then added. The suspension was left at room temperature $\left(22^{\circ} \mathrm{C}\right)$ for $80 \mathrm{~min}$ with occasional shaking, and then the spores were washed four or five times by centrifuging at $3000 \mathrm{rev}$. $\mathrm{min}^{-1}$ for $5 \mathrm{~min}$ and resuspended in sterile water. After the final wash, the mutagenized spores were heat-shocked for $5 \mathrm{~min}$ at $48^{\circ} \mathrm{C}$, and plated at $10^{8}$ spores per plate on complete media to select viable spores. (Of the $10^{8}$ spores plated, about $10^{4}$ spores survive NTG mutagenesis). Spore isolation and collection techniques were as previously described (Keyhani, Keyhani \& Goodgal, 1972).

Replica-plating of PII. Strain PI I is a colonial mutant which forms dwarf sporangiophores approximately I to $2 \mathrm{~mm}$ long and $0.05 \mathrm{~mm}$ wide. In contrast to the wild-type sporangiophore, the dwarf sporangiophore does not taper up to the sporangium base and the sporangium is relatively small (about $0.2 \mathrm{~mm}$ diam.). Replicas were formed by making a print with velvet of spaced dwarf colonial mutants when sporangia had clearly formed. Spores transferred by this method gave colonies in exactly corresponding positions. After several days, comparison between replicas and the original plate allowed possible auxotrophs to be identified, and tested further. Up to six clear replicas could be made from one velvet allowing a check for several growth requirements at once. The limiting factor in Phycomyces is that only the dwarf colonial strain can be replicaplated easily. By lowering the $\mathrm{pH}$ of the media (to $\mathrm{pH} 3 \cdot 2$ ) Phycomyces will grow colonially (S. Park, unpublished results.) However, wild-type sporangiophores are long and not suitable for replica-plating.

\section{RESULTS AND DISCUSSION}

For organelle transfer studies in Phycomyces (Goodgal et al., 1975), drug-sensitive recipient strains are needed so that any growth of progeny in the presence of a drug might be attributed to transfer of the drug resistance marker from the donor organelle. Strain $P 8$ has a high level of resistance to erythromycin; growth is slower at $5 \mathrm{mg}$ erythromycin $\mathrm{ml}^{-1}$ and complete inhibition only occurs at concentrations greater than $8 \mathrm{mg} \mathrm{ml}^{-1}$. The isolation of 
mutants more sensitive to erythromycin was therefore considered desirable and a procedure was devised for selecting mutants which showed complete inhibition of growth at $5 \mathrm{mg}$ erythromycin $\mathrm{ml}^{-1}$.

Some variation in erythromycin sensitivity was noted between different clones of $P 8$. One $P 8$ kanamycin-sensitive strain was inhibited by $5 \mathrm{mg}$ erythromycin $\mathrm{ml}^{-1}$ for 2 days and then growth proceeded normally. As erythromycin breaks down chemically, no results of growth after 4 days were used in determining drug sensitivity. The original P8 auxotrophic strain is referred to as normal P8 throughout this paper to distinguish it from P8 drugsensitive strains.

\section{Differential heat selection}

Mycelia and germinating spores die at temperatures above $35^{\circ} \mathrm{C}$, whereas ungerminated spores can withstand such temperatures for extended periods. Normal $\mathbf{P} 8$ spores were mutated, heat-shocked and aerated at $10^{6}$ spores per culture in a minimal medium liquid culture with $5 \mathrm{mg}$ erythromycin $\mathrm{ml}^{-1}$. Three cycles of liquid culture-filtration followed by plating of surviving spores on minimal medium resulted in germination of over 200 spores. These were separated by subculturing each spore on to a small agar dish. After sporangia were generated, a single sporangium from each dish was tested for erythromycin sensitivity. Only four out of 200 sporangia showed drug sensitivity. Spores from these four sporangia were plated on minimal agar and separated on to individual dishes after germination. Sporangia arising from these dishes were examined for erythromycin sensitivity. None of over 100 progeny sporangia tested showed significant erythromycin sensitivity. A further selection step seemed necessary to enrich for more stable drug-sensitive mutations.

Pieces of dialysis tubing were cut to the size of a Petri dish, boiled for $10 \mathrm{~min}$ and then placed on minimal medium agar plates either with erythromycin $\left(5 \mathrm{mg} \mathrm{ml}^{-1}\right)$ or without erythromycin (control). The dialysis tubing allowed the rapid transfer of spores to new nutrient dishes. Mutated $\mathbf{P} 8$ spores from the liquid culture-filtration selection were heatshocked and plated at $5 \times 10^{3}$ spores per plate on dishes covered with dialysis tubing. Spores were allowed to germinate and to begin spreading a thin mycelial cover over the plate. Plates were then placed at $37^{\circ} \mathrm{C}$ for 16 to $24 \mathrm{~h}$ to kill germinated spores and mycelia. After this time the dialysis tubing from the erythromycin plate was transferred to another erythromycin plate and dialysis tubing from the control plate was transferred to a plate without erythromycin. These plates were left at room temperature for several days and then the procedure was repeated. After a second treatment at $37^{\circ} \mathrm{C}$, the dialysis tubing from selection plates and control plates was transferred to minimal medium agar plates without erythromycin. Previously ungerminated drug-sensitive spores survived heat treatment and grew. On the control plate, all viable spores had germinated and had subsequently been killed by the heat treatment. Plates left at room temperature and never exposed to $37^{\circ} \mathrm{C}$ grew normally with about $90 \%$ of plated spores germinating.

Spore germination counts were necessary to estimate spore viability and the percentage of erythromycin-sensitive mutants. In order to obtain colonial growth to facilitate counting, spores were tested on minimal medium acid plates ( $\mathrm{pH}_{3 \cdot 2}$ ) without erythromycin after the last heat treatment. When compared to growth of spores on minimal medium at neutral $\mathrm{pH}$, the viability of erythromycin-sensitive spores on acid plates was too low to use this technique (about $70 \%$ fewer spores germinated at $\mathrm{pH} 3.2$ than at $\mathrm{pH} 7 \cdot 0$ ). However, by using low dilutions of spores on minimal medium agar plates and observing germination at $16 \mathrm{~h}$, spore germination counts could be determined. The addition of $3 \%(\mathrm{v} / \mathrm{v})$ ethanol to complete and minimal medium agar plates induced colonial growth in mycelia without significantly decreasing spore viability (less than $5 \%$ decrease).

To ensure spore germination could not take place at $37{ }^{\circ} \mathrm{C}$, normal $\mathrm{P} 8$ spores were heatshocked, plated and immediately placed at $37^{\circ} \mathrm{C}$ for $20 \mathrm{~h}$ on minimal medium agar dishes (with nicotinic acid). No germination occurred during this period. After return to room temperature, about $80 \%$ of the spores germinated. 
Using the conditions described to maximize survival of erythromycin-sensitive spores, mutagenized P8 spores were subjected to two cycles of differential heat selection. Ungerminated spores were plated on complete medium, allowed to generate sporangia and then these sporangia were examined for drug sensitivity. Twenty-four sporangia from about 40 spores surviving heat selection were tested; all grew slower at $5 \mathrm{mg}$ erythromycin $\mathrm{ml}^{-1}$. In the next generation, progeny derived from 14 of the original 24 sporangia showed continued sensitivity to $5 \mathrm{mg}$ erythromycin $\mathrm{ml}^{-1}$. After three generations, progeny spores descended from three of the original 24 sporangia tested grew slower at $5 \mathrm{mg}$ erythromycin $\mathrm{ml}^{-1}$. Progeny spores were examined at low dilution or on ethanol plates so that the percentage of erythromycin-sensitive spores could be estimated (Table I).

One variation of this procedure involved heat selection while the spores were in liquid culture (Table I). Mutated P8 spores from one cycle of liquid culture-filtration selection in the presence of erythromycin were heat-shocked and $10^{3}$ spores were added to liquid culture without erythromycin and immediately placed at $37^{\circ} \mathrm{C}$ for $16 \mathrm{~h}$. This step eliminated prototrophic germlings which were not filtered out in the previous selection step. After $16 \mathrm{~h}$, the culture was aerated at room temperature allowing germination of erythromycin-sensitive spores. After a second heat selection cycle, the five spores that germinated in the minimal medium liquid culture (without erythromycin) were isolated and examined for erythromycin sensitivity on agar plates. Two mutants were completely inhibited at $5 \mathrm{mg}$ erythromycin $\mathrm{ml}^{-1}$ while one mutant showed total inhibition at $4 \mathrm{mg} \mathrm{ml}^{-1}$. Testing the progeny of these mutants revealed continued sensitivity as low as $3 \mathrm{mg} \mathrm{ml}^{-1}$ - at this concentration initial germ tubes were formed but extended poorly. In additional tests on progeny, erythromycin sensitivity remained at this level.

Less stable drug-sensitive mutants found in early experiments showed initial sensitivity that disappeared over several generations. These mutations could possibly be cytoplasmic in nature, explaining their disappearance after several progeny generations on complete media where wild-type mitochondrial multiplication might be favoured.

\section{Auxotrophic mutants from strain PI I}

The dialysis tubing and differential heat treatment combined with replica-plating of the dwarf colonial strain PI I offers a rapid selective method for isolating and characterizing auxotrophic mutants. Those spores which failed to germinate on minimal medium agar plates were plated on complete medium, allowed to germinate, subcultured and spaced (40 per plate) in preparation for replica-plating.

Liquid culture growth of PII was extremely slow so that liquid culture-filtration was not used as an initial selection device as with strain P8. Strain PI I was mutated, grown on complete medium, collected and plated at $10^{4}$ spores per plate on minimal medium agar covered with dialysis tubing. These spores were grown at room temperature for 2 to 4 days or until small colonies of thin mycelia appeared. The plates were then placed at $37^{\circ} \mathrm{C}$ for $16 \mathrm{~h}$. After this period they were returned to room temperature for 2 to 4 days and then the procedure was repeated. After the final heat treatment, the dialysis tubing was transferred to complete medium where auxotrophic spores germinated (Fig. I). PI I spores were more heat-sensitive than wild-type spores and no auxotrophic spores survived incubation at $37^{\circ} \mathrm{C}$ for longer than $24 \mathrm{~h}$. Only $20 \%$ of the ungerminated spores survived $16 \mathrm{~h}$ incubation at $37{ }^{\circ} \mathrm{C}$. We tried various methods of maximizing survival of PI I spores at $37^{\circ} \mathrm{C}$, and found that survival increased twofold if water agar was substituted for minimal medium during the $37^{\circ} \mathrm{C}$ treatment. Although the spore survival was greater after $\mathrm{I} 6 \mathrm{~h}$ incubation at lower temperatures $\left(50 \%\right.$ survival at $30{ }^{\circ} \mathrm{C}$ and $90 \%$ survival at $\left.25{ }^{\circ} \mathrm{C}\right)$, temperatures of 35 to $37{ }^{\circ} \mathrm{C}$ were necessary to kill germinating spores and mycelia. For strain PI I, maximal conditions for auxotrophic selection were $16 \mathrm{~h}$ at $37^{\circ} \mathrm{C}$ on water agar plates.

Using the heat selection technique, over 50 auxotrophs were isolated, many with multiple markers. Seven have been fully characterized (mutant strains P55 to P6I). Mutant strain 


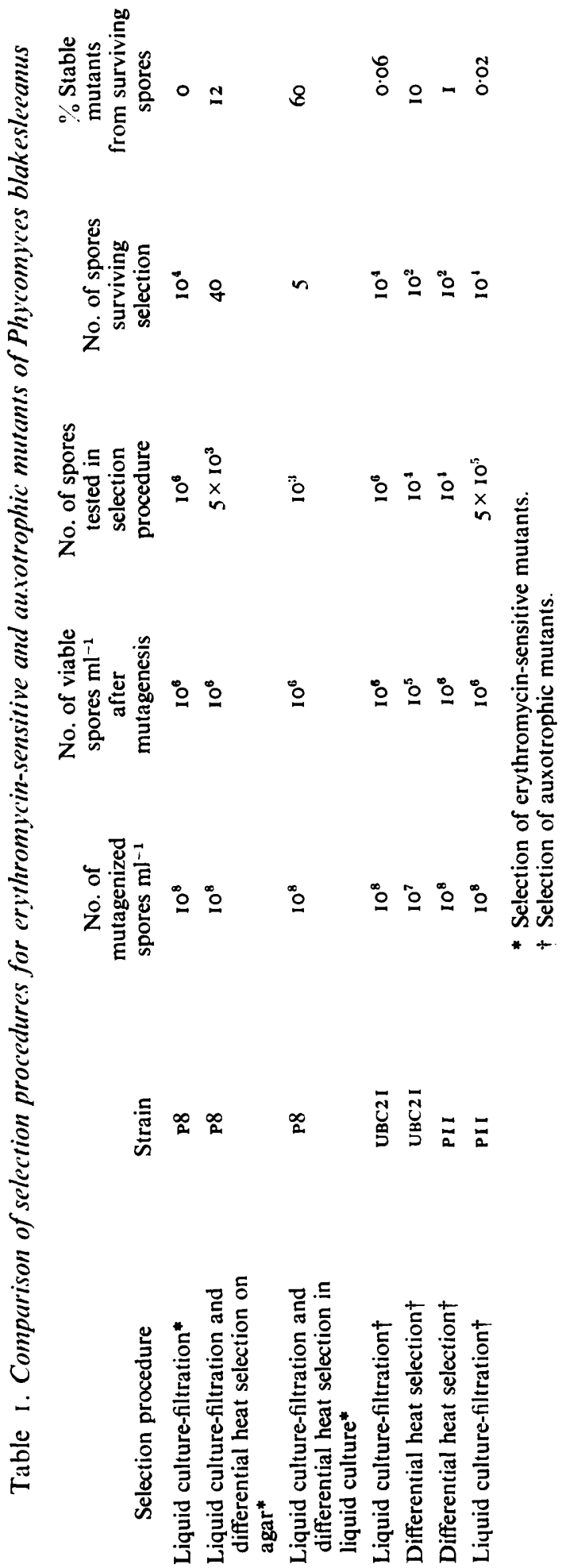




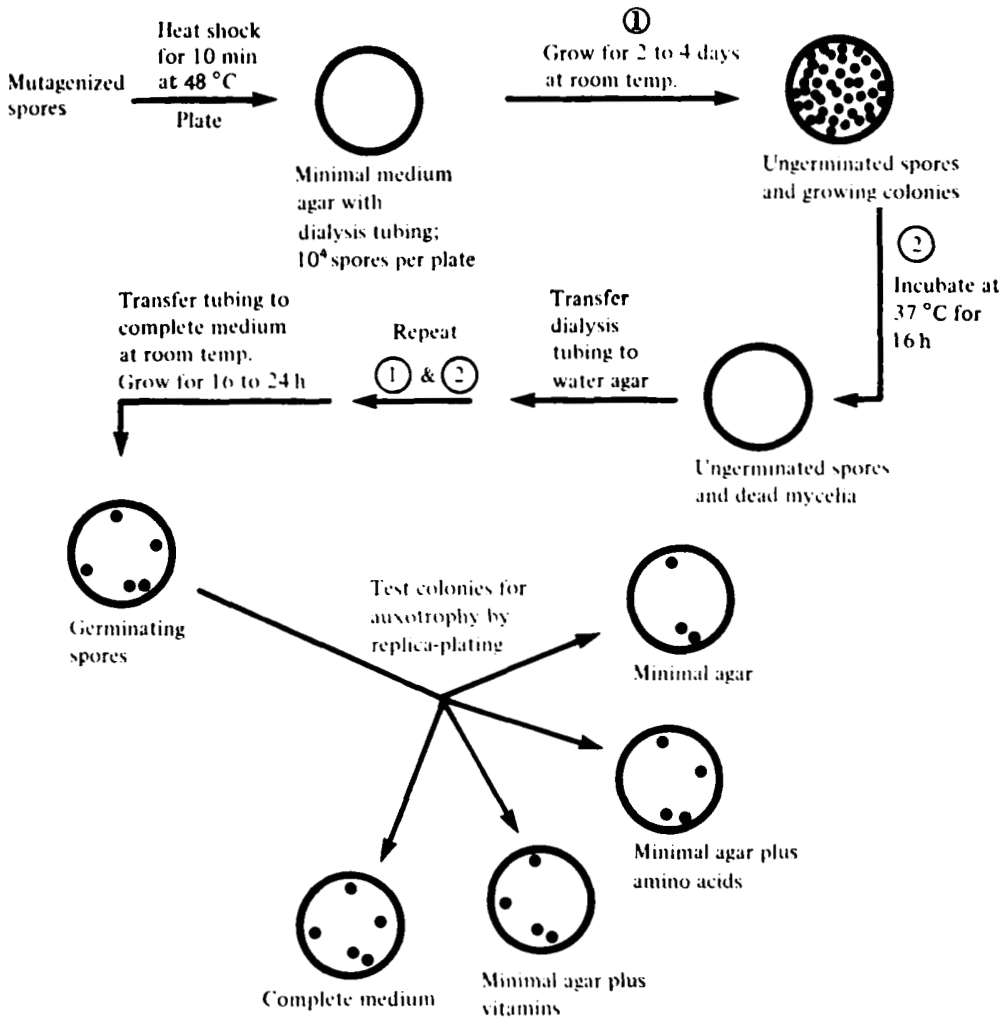

Fig. I. Method of differential heat selection with Phycomyces blakesleeanus strain PI I.

P6I, originally observed as a folic acid auxotroph, has been shown to require $p$-aminobenzoic acid. Replica-plating has facilitated the characterization of these auxotrophic mutants.

Previous methods for auxotrophic enrichment with Phycomyces have at best enabled auxotroph isolation in $0.066 \%$ of selectively enriched spores (about one auxotroph in 1500 spores selected by liquid culture-filtration). The observation that mycelial death occurs in I $6 \mathrm{~h}$ at temperatures above $35^{\circ} \mathrm{C}$ while ungerminated spores can survive extended periods at 35 to $37^{\circ} \mathrm{C}$ has enabled us to devise an enrichment procedure resulting in isolation of one stable auxotroph for every 75 to 100 mutagenized spores examined or greater than $1 \%$ of colonies surviving heat selection. Starting with $10^{4}$ mutagenized spores plated, about 100 spores survive heat selection and I \% of these are stable auxotrophic mutants. This represents an auxotrophic selection frequency of $0.01 \%$. This frequency would be expected to be at least twofold greater in the wild-type strain where spore survival after heat treatment is $80 \%$ compared with $40 \%$ for strain PI I. Indeed, current work with wild-type strain UBC2 I shows a I0-fold increase of stable auxotrophs surviving heat selection (Table I) as might be predicted from the results of erythromycin sensitivity enrichment in strain P8. (Two nicotinic acid auxotrophic mutants have been characterized so far in strain UBC2I.) Strain PI I grows poorly in liquid culture, making liquid culture-filtration selection impractical, so that differential heat selection offers the first effective method of enrichment for auxotrophs in this strain.

Our thanks to M. Kahn, G. Simms and S. Park for their valuable help and advice. One of us (K.J.B.) is supported by a National Institutes of Health Pre-doctoral Training Grant (PHS-I-T32-GMo7229). 


\section{REFERENCES}

Goodgal, S. H., Kahn, M. \& Keyhani, J. (1975). Moat, A. G., Peiers, N., JR \& Srb, A. M. (1959). The transfer of biological properties of Phycomyces blakesleeanus by isolated mitochondria. Biophysical Journal 15, Biophysical Society Abstracts, 19a.

HenRY, S. A. (1973). Death resulting from fatty acid starvation in yeast. Journal of Bucteriology I16, I 293-I 303.

Keyhani, J., Keyhani, E. \& Goodgal, S. H. (1972). Studies on the cytochrome content of Phycomyces spores during germination. European Journal of Biochemistry 27, 527-534.

LederberG, J. \& LederberG, E. M. (1952). Replica plating and indirect selection of bacterial mutants. Journal of Bacteriology 63, 399-406.

Megnet, R. (1964). A method for the selection of auxotrophic mutants of the yeast Schizosaccharomyces pombe. Experientia 20, 320-321.

Selection and isolation of auxotrophic yeast mutants with the aid of antibiotics. Journal of Bacteriology 77, 673-677.

Ridgway, G. J. \& Douglas, H. C. (1958). Unbalanced growth of yeast due to inositol deficiency. Journal of Bacteriology 76, 163-166.

SNow, R. (1966). An enrichment method for auxotrophic yeast mutants using the antibiotic nystatin. Nature, London 211, 206-207.

Woodward, V. W., De Zeeuw, J. R. \& SRB, A. M. (1954). The separation and isolation of particular biochemical mutants of Neurospora by differential germination of conidia, followed by filtration and selective plating. Proceedings of the National Academy of Sciences of the United States of America 40, 192-200. 